\title{
Wie und warum Staat Sicherheit produziert \\ Dargestellt anhand der Versicherheitlichung grenzüberschreitender Mobilität seitens der EU und der so produzierten Räume des Risikos
}

\section{Bernd Belina, Frankfurt am Main}

Der grundlegende Umbau des Grenzregimes der Europäischen Union (EU) im Rahmen der Vergemeinschaftung der Grenz- und Migrationspolitik, der entlang der Aussengrenze ebenso wie inner- und ausserhalb des EU-Territoriums neue Räume produziert, ist ganz an der Herstellung von «Sicherheit» ausgerichtet (vgl. Andreas 2003; Belina \& MiggelbrinK 2010; BUCKEL \& WisSEl 2009; EIgMÜLler 2007; HUYSmans 2000; Raj 2006; Transit Migration ForschungsgrupPe 2007; Walters 2006). Dabei gilt die zentrale Einsicht der Debatten um «Sicherheit» (Dalby 1997), «Versicherheitlichung» (Buzan, Wæver \& De Wilde 1997) und die «Sicherheitsgesellschaft» (SINGELNSTEIN \& STOLle 2008): Werden soziale Phänomene als Sicherheitsprobleme diskutiert und behandelt, wird von ihrer umkämpften Produktion abstrahiert, wodurch sie depolitisiert und bestehende Herrschaftsverhältnisse legitimiert werden (vgl. Neocleous 2008). Dieses Argument will ich im Folgenden mit Bezug auf FoucAult entwickeln, über dessen Konzeption von «Sicherheit» mit der materialistischen Staatstheorie hinausgehen und sie zudem vertiefen, indem ich am zur Illustration herangezogenen Beispiel der EU-Aussengrenze der Frage nachgehe, welche Rolle Raumproduktionen in der Sicherheitslogik spielen.

\section{Foucault als poststrukturalistische Antithese zum Marxismus?}

Nachdem Foucault v.a. von Marxist/inn/en dafür kritisiert wurde, in seinen früheren Studien zur «Mikrophysik der Macht» den Staat ausgeblendet zu haben, wendet er sich diesem in den Vorlesungen der Jahre 1977-79 explizit zu (GoRdon 1991: 4). Über die in «Überwachen und Strafen» (Foucault 1994) entwikkelte Unterscheidung von juridischer und Disziplinarmacht hinausgehend gilt seine Suche nunmehr einer «Gesamttechnologie der Macht» (Foucault 2004a: 180).

Aus der angloamerikanischen Debatte sind es Geograph/inn/en gewohnt, sich Foucault als «Poststrukturalisten» vorzustellen, für den es «no extra discursive 〈real〉 outside of cultural systems» (PRATT 1994: 468) gebe, und der deshalb mit den vermeintlich naiven Annahmen des historischen Materialismus bezüglich der sozialen Wirklichkeit gebrochen habe. Im Gegensatz dazu folge ich der Einschätzung, nach der bei FouCAULTS Thematisierung der Biomacht, also beginnend mit «Der Wille zum Wissen» (Foucault 1997) und dann insbesondere in den Gouvernementalitätsvorlesungen (Foucault 2004a, 2004b), «Konvergenzen mit [...] marxistischen Positionen» (JEssop 2007b: 67) auszumachen sind (vgl. LeMKe 1997: 142). Insbesondere nähert sich Foucault den Debatten der marxistischen Staatstheorie an, in denen zu dieser Zeit GramsCI (wieder-) entdeckt wird (vgl. KRAMER 1975: 65).

GramsCi betont, dass man «unter dem Staat ausser dem Regierungsapparat auch den 〈privaten〉 Hegemonieapparat oder Zivilgesellschaft verstehen muss» (1992: 816), also z.B. Schulen, Kirchen, Medien. Den Staat i.e.S. und die Zivilgesellschaft zusammen bezeichnet er als «integralen Staat», wobei Ersterer in der Herrschaftsausübung auf «Zwang» (ebd.: 783) zurückgreifen kann, während Letztere die Individuen durch «Hegemonie» (ebd.) dazu erzieht, «sich in einer dem Staat gemässen Weise selbst zu regieren» (BUCKEL \& FisCHER-LESCARNo 2007: 12). Diese Herrschaft durch Zustimmung ist umkämpft, weshalb der je konkrete integrale Staat aus einem «Prozess sich ständig verschiebender und ändernder Kompromissgleichgewichte» (Demirović 2007: 32) resultiert.

Auf diese Erweiterung des Staatsbegriffes im Marxismus bauen unter den Zeitgenossen Foucaults etwa Althusser (1977) und Poulantzas (2002) auf. Insbesondere die Staatstheorie von Poulantzas, in der er den Staat als soziales Verhältnis und materielle Verdichtung gesellschaftlicher Kräfteverhältnisse bestimmt, enthält «zahlreiche Anleihen und Affinitäten» (JESSOP 2007a: 56) von bzw. mit GramsCI und stellt zudem einen ersten Versuch «einer materialistischen Aneignung von Foucault» (Lindner 2006: 154) dar (vgl. Jessop 2007b: 59f.). So bezieht sich PoulantZAS positiv auf «Überwachen und Strafen» (Foucault 1994), in dem er einen Aspekt der Individualisierung durch den Staat treffend beschrieben sieht, und betont, dass der Staat «zur Produktion dieser Individualität durch eine Gesamtheit von Wissenstechniken (Wissenschaft) und Machtpraktiken bei[trägt]» (PoulantZAS 2002: 93). Auch das Verständnis von Macht beider Autoren weist Gemeinsamkeiten auf. Nach PoulantZAS ist sie «weder Quantität noch Besitztum [...] und auch keine an das Wesen einer Klasse [...] gebundene Eigenschaft» (ebd.: 177), und er betont: «Das Macht- 
feld ist [...] strikt relational» (ebd.: 178). Folglich ist der Staat für ihn kein Machtzentrum an sich, sondern nur «ein Ort und ein Zentrum der Machtausübung, [er] besitzt jedoch selbst keine eigene Macht» (ebd.: 179). Auch Foucault (1997: 113-118) bestimmt Macht durch Kräfteverhältnisse und Relationalität, und er lehnt es ab, sie gleichzusetzen mit «Regierungsmacht, als Gesamtheit der Institutionen und Apparate, die die bürgerliche Ordnung in einem gegebenen Staat garantieren» (ebd.: 113). Gleichwohl betont auch er, dass die Strategien der Macht sich «in den Staatsapparaten, in der Gesetzgebung und in den gesellschaftlichen Hegemonien verkörpern» (ebd.: 113f.). In FoucAults Befassung mit dem Staat tauchen «poulantzianische Gedankenfiguren auf» (LindNER 2006: 154), und er spricht ihm «eine privilegierte Rolle als dem Ort der strategischen Kodierung der Pluralität der Machtverhältnisse zu» (Jessop 2007b: 79). Auf eine zentrale Kritik Poulantzas' (2002: 179) an Foucault wird im Folgenden näher eingegangen: «Die Frage welche Macht und wozu diese Macht scheint bei ihm völlig ihre Gültigkeit verloren zu haben». Hier sehe ich die zentrale Notwendigkeit einer materialistischen Erdung von FouCAULTs Staatsverständnis.

\section{Regieren und Biomacht}

Foucaults Thematisierung des Staates geschieht über den «Schlüsselbegriff» (LEMKE 1997: 150) des «Regierens», womit er die Führung sowohl des Selbst als auch Anderer bezeichnet, die ein spezifischer Typus der Macht leistet, die Biomacht. Neben der juridischen und der Disziplinarmacht identifiziert Foucault mit dieser eine dritte, die beiden erstgenannten weitgehend integrierende Form der Macht (vgl. LemKe 1997: 188-194). Während die juridische Macht Rechtssubjekte anhand des Gesetzes sortiert, und die Disziplinarmacht sie als Subjekte hervorbringt (Foucault 1994), richtet die Biomacht «ihre Zugriffe auf das Leben und seinen ganzen Ablauf» (FoucAult 1997: 165), auf «die sorgfältige Verwaltung der Körper und die rechnerische Planung des Lebens» (ebd.: 167). Gegenstand der Biopolitik «sind die einer Bevölkerung eigenen Massenphänomene, die Bedingungen ihrer Variation, die Kontrolle von Wahrscheinlichkeiten, die Modifikation ihrer Effekte» (LemKe 1997: 136), ihre Mittel sind «Regulierung und Kontrolle» (ebd.: 126) und ihr Ort ist der Staat (ebd.: 137).

Foucault diskutiert die drei Logiken der Macht auch mit Bezug auf je unterschiedliche «Raumfragen» (2004a: 27), also den unterschiedlichen, aus ihrer Anwendung resultierenden Raumproduktionen. Auch wenn er es «versäumte, eine praktikable kritische Theorie dessen zu entwickeln, worum es sich bei Raum und Zeit handeln könnte» (Harvey 2007: 46; Über- setzung fremdsprachiger Zitate B.B.), kann an diese Überlegungen angeschlossen werden, um in Abschnitt 5 zu fragen, welche Art von Räumen, welche «Sicherheitsräume» (Foucault 2004a:26) in und aufgrund der Sicherheitslogik produziert werden.

Foucault spricht von der Biomacht, weil ihr Hauptgegenstand, die Bevölkerung, wegen ihrer Naturalität, Relativität und Komplexität nicht vollständig gesteuert, sondern nur durch Beeinflussungen nach Massgabe der in ihr geltenden natürlichen oder quasinatürlichen Spielregeln «von aussen» gelenkt werden kann, in der Hoffnung, über «das Spiel dieser Faktoren effektiv auf die Bevölkerung ein[zu]wirken» (FAucoult 2004a: 110). Dieses governing at a distance (GARLAND 1996) verzichtet in vielen Bereichen auf den direkten Zugriff auf Individuen zugunsten einer indirekten Verwaltung von Gruppen, Aktivitäten und Räumen (MOPAS \& VALVERDE 2004: 246).

Für diese Art des Regierens von Bevölkerung ist ein umfangreiches Wissen über diese vonnöten. Erstens generiert die «Staatswissenschaft» (PoulantZas 2002: 84) im Sinne und nach den Massgaben des Staates ein Wissen über die Zusammenhänge, die das komplexe Ganze der Bevölkerung strukturieren, über die internen und quasi-natürlichen Gesetze ihres Funktionierens. Dank ihres bornierten Standpunkts, der ganz dem Mittun beim «Regieren» der Bevölkerung auf der Basis der eingerichteten Spielregeln verpflichtet ist, genügt es solchen Wissenschaften üblicherweise, von der «Abstraktion» (MARX 1969: 631) namens «Bevölkerung» (ebd.) auszugehen und mit einer «chaotische[n] Vorstellung des Ganzen» (ebd.) zu operieren, die die Fetischisierungen durch Kapital-, Staatsund Rechtsform gerade nicht zu kritisieren vermag, sondern theoretisch reproduziert. Zweitens produzieren die Staatsapparate mittels Statistiken - dem «Wissen des Staates über den Staat» (Foucault 2004a: 455) - alle möglichen Daten über die Bevölkerung, um die von der Wissenschaft produzierten Modelle und «Gesetze» des Funktionierens der Bevölkerung gouvernemental auch tatsächlich anwenden und ins Werk setzen zu können.

Während für die Disziplinarmacht ein möglichst genaues Wissen über das Individuum und seine «Seele» vonnöten ist, um auf dieses direkt Einfluss nehmen und es formen zu können, braucht es für das indirekte Führen der Biomacht aggregierte Daten über eine Population, entweder über die Bevölkerung als Ganze oder über Untergruppen, die auf Basis der «Gesetze» der Bevölkerung gebildet werden. Gleichwohl kommen Individuen in dieser Logik vor, und zwar als zu identifizierende und in diesem Prozess zu konstituierende. Zum einen müssen, um die betrachtete Population klar abzugrenzen, Individuen anhand 
von Merkmalen als ihr zugehörig oder nicht zugehörig bestimmt werden. Dabei setzt sich mit der «Tyrannei des Nationalen» (NoIRIEL 1994) im 19. Jahrhundert die Vorstellung und Sortierung der «Bevölkerung» als Staatsvolk und Nation durch. Zum anderen wird bei der Umsetzung der gouvernementalen Logik notwendig auf Individuen zugegriffen, die als solche identifizierbar sein müssen (vgl. Legnaro 2008).

\section{Zwischenbilanz: wie / warum?}

Bei den Begriffen Gouvernementalität und Biomacht steht das «wie» von Macht und Regierung im Zentrum, «[g]overnmentality is about how to govern» (GoRDON 1991: 7; Hervorhebung B.B.). Foucault benutzt zahlreiche Beispiele, anhand derer er seine Thesen entwikkelt, vor allem Dokumente, in denen über die Regierungskunst räsoniert wird. Immer wieder jedoch geht er über die Analyse von Texten hinaus und belässt es nicht bei der Untersuchung des Wissens über das Regieren, sondern setzt dieses Wissen ins Verhältnis $\mathrm{zu}$ einer praktisch hergestellten gesellschaftlichen Realität, die sich nicht auf Text reduzieren lässt, und deren «Elemente» es mittels dieses Wissens «wechselseitig in Gang zu setzen [gilt]» (2004a: 101). So betont er bezüglich der Wechselbeziehungen in einem von ihm diskutierten Buch aus dem 15. Jahrhundert, dass diese «sich in der Wirklichkeit orten [lassen]» (ebd.: 151) und an anderer Stelle: «Die kapitalistische Ausbeutung ist in gewisser Weise realisiert worden, ohne dass man jemals ihre Theorie wirklich in einem Diskurs formuliert hätte» (Foucault 2002: 783). Ganz ähnlich in «Der Wille zum Wissen»:

«Die Abstimmung der Menschenakkumulation mit der Kapitalakkumulation, die Anpassung des Bevölkerungswachstums an die Expansion der Produktivkräfte und die Verteilung des Profits wurden auch durch die Ausübung der Bio-Macht in ihren vielfältigen Formen und Verfahren ermöglicht» (Foucault 1997: 168).

Hier betrachtet Foucault das Aufkommen der Biomacht also im Zusammenhang mit der gesellschaftlichen Entwicklung der Produktivkräfte, die nicht in einem engen Diskursbegriff (i.S. von Text) aufgeht. Während er sich von einer Vorstellung abgrenzt, die jede Wissensproduktion aus einer singulären Ursache oder einer Geschichtsphilosophie ableiten will (BALIBAR 1991; Demirović 2008), ist er zugleich weit davon entfernt, ein je «in seiner Positivität» (Foucault 1992: 34) zu untersuchendes Verhältnis gesellschaftlich gültigen Wissens mit sozialen Prozessen, die nicht auf diskursive Praktiken zu reduzieren sind, zu leugnen. Im Gegenteil fordert er auf zur Analyse der «Interaktionen und [...] zirkulären Aktionen» (ebd.: 37), der «nichts fremder [ist] als die Verwerfung der Kausalität» (ebd.). Hierzu will er die «Erscheinungsbedingungen einer Singularität in vielfältigen bestimmenden
Elementen ausfindig [...] machen» (ebd.). Nach BALIBAR (1991: 58) ist dieses Vorgehen

«in keiner Weise unvereinbar mit den von Marx praktizierten strategischen Analysen, und sie widersprechen auch nicht der Idee der 〈Überdeterminierung〉, derer sich Althusser [...] bedient hat».

Auch LiNK (2008) betont die positive Bezugnahme Foucaults auf Althusser und insbesondere MarX, die nach dem «spektakuläre[n] Widerruf» (ebd.: 444) seiner frühen Polemiken gegen den vermeintlich geschichtsphilosophisch-naiven MARX zu beobachten ist. Foucault betont lediglich, dass die

«Funktionalität des Wissens für die Macht stets als Resultat (ex post) «strategischer> Prozesse zu begreifen und nicht als Sich-Durchsetzen eines (ex ante bereits alle Wissensproduktion bestimmenden) Klasseninteresses innerhalb der Diskurse [ist]» (ebd.: 450).

Diesen Prozess, der «Individuen und Gesellschaften einen 〈Willen zur Normalität〉 [implementiert], der die Körper prägt und der gerade auch ökonomisch wirksam ist» (ebd.: 452), bezeichnet LiNK als «Normalismus».

$\mathrm{Zu}$ dem «wie» von Gouvernementalität und Biomacht kommen also auch bei Foucault Andeutungen eines «warum», einer Erklärung, nicht nur ihrer Funktionsweise, sondern auch ihrer Funktionen. An diese Hinweise anschliessend gilt es, die Untersuchung des «how to govern» (GoRdon 1991: 7) zu ergänzen mit dazugehörigen «answers to the 〈why questions» (Hudson 2006: 385), die die Kritik der Politischen Ökonomie liefert, und die erklären können, «welche Macht und wozu diese Macht» (PoulantZas 2002: 179) relevant wird. Hierzu bedarf es m.E. einer «Erdung» FoucAults mittels materialistischer (Staats-) Theorie. Gesellschaftliche Phänomene gilt es - mit FoucaulT - zu untersuchen im Hinblick auf ihre «Funktion in Bezug auf die Lenkung, Führung und Anordnung von Menschen» (Füller \& Marquardt 2009: 93). Dabei gilt es - anders als Foucault -, nicht selbst in eine Geschichtsphilosophie zu verfallen, die «die Begriffe der Gesellschaft als auch der Politik universalisiert und ontologisiert» (Demirović 2008: 191), und seinen Hang zum Begründungsrelativismus zu meiden, der unmittelbar zum performativen Widerspruch führt (LINDNER 2008: 210f.).

\section{Sicherheit und Risiko}

Warum ausgerechnet «Sicherheit» zum «Hauptziel der Gouvernementalität» (Foucault 2004a: 506; vgl. Purtschert, Meyer \& Winter 2008) avanciert, kann m.E. von bzw. nur mit Foucault nicht beantwortet werden. Während GidDENs (1996) für Unsicherheit die Reflexivität der Moderne verantwortlich macht, oder Bauman (1997) sie auf die Sterblichkeit des Menschen 
zurückführt, scheint es mir mit NEOCLEOUs (2000a: 59) sinnvoll, die Thematisierung von Sicherheit als notwendige Begleiterscheinung des auf Privateigentum basierenden Kapitalismus zu verstehen:

«Die Sicherheit ist der höchste Begriff der bürgerlichen Gesellschaft [...], dass die ganze Gesellschaft nur da ist, um jedem ihrer Glieder die Erhaltung seiner Person, seiner Rechte und seines Eigentums zu garantieren» (MARX 1970: 365f.).

Diese Sicherheit ist andauernd Risiken ausgesetzt, weil der Wert des Eigentums als soziales Verhältnis der steten Gefahr der Entwertung ausgesetzt ist, auf die der/die Einzelne keinen Einfluss hat. Aus diesem im Kapitalismus eingerichteten Verhältnis, so Neocleous (2000a: 45-91), gilt es, die daraus abgeleiteten Risiken, die der Staat als bekämpfenswert erachtet, zu erklären. Dabei kann es sich ganz unmittelbar um Risiken für konkrete Eigentumswerte handeln, viel öfter aber um solche für die sozialen Formen Staat, Nation und Recht (und Ordnung), die zur Absicherung einer auf dem Privateigentum an Produktionsmitteln basierenden Wirtschaftsweise notwendig sind. Erst auf der Basis dieses Hinweises auf das «warum» der Herstellung von Sicherheit durch den Staat wird bezüglich der Biomacht erklärbar, «wozu diese Macht» (Poulantzas 2002: 179) taugt, die sich durch die Berechnung des Eintritts von Schadenswahrscheinlichkeiten, mithin durch Risikokalkulation, auszeichnet.

Dabei ist nicht mehr die Norm der Ausgangspunkt, sondern «das (empirisch) Normale» (LemKe 1997:190), das statistisch ermittelt und dabei anhand der in die Statistik eingehenden Vorannahmen produziert wird. Die Berechnung des Risikos der Abweichung hiervon erlaubt «die Unterscheidung von 〈gefährdeten〉 bzw. 〈gefährlichen〉 Individuen und Klassen» (LEMKE 2000: 35). Indem Letztere als Verursacher/innen zukünftiger Schäden behandelt werden, begründet die Risikoberechnung anhand von «Risikofaktoren» (vgl. SINGELNSTEIN \& STOLLE 2008: 33f.) ein «spezifisches Verhältnis zur Zukunft» (Aradau \& VAN Munster 2007: 97) und Handlungsanweisungen für den Staat. Auch wenn die Risikoberechnung sich überhaupt nicht für Individuen interessiert (vgl. EwaLD 2002), liegt ihr ganzer Zweck darin, ihre Ergebnisse als staatliche Kontrollpolitik auf Individuen anzuwenden. Auf der einen Seite der eingezogenen Grenze wird der Staat das Verhalten «beeinflussen, anreizen» (Foucault 2004a: 506) und den Subjekten «Freiräume des Handelns» (SINGELNSTEIN \& STOLLE 2008: 70) zugestehen, in denen subtile Anreize dafür sorgen, dass sie sich konform verhalten wollen. Mit MARTI (2008:300) wäre «die ungleiche Verteilung ökonomischer Ressourcen» als Erklärung des Willens zum Mittun zu ergänzen, mithin das «warum» des «wie».

Auf der anderen Seite der eingezogenen Grenze werden diejenigen, die als riskant gelten, präventiv behandelt, bevor sie etwas Unerwünschtes tun können. Das Problem, dass man nie wissen kann, wer genau sich wann welcher Abweichung von der gewünschten Ordnung schuldig machen wird, wird durch die Berechnung der Wahrscheinlichkeit zukünftiger Abweichungen technisch gelöst. Welche an den die «Population» konstituierenden Individuen abstrahierten Merkmale in welcher Weise in die Risikokalkulation eingehen, ist dabei immer eine politische Entscheidung, weil aus Erfahrungswerten auf der Basis aggregierter Daten niemals auf das zukünftige Verhalten Einzelner geschlossen werden kann. Das Problem des «ökologischen Fehlschlusses» (RoBINson 1950) wird mithin politisch gelöst, indem seine Unschärfe billigend in Kauf genommen wird.

Diese Umgangsweise mit Abweichler/innen widerspricht der Behauptung einiger Gouvernementalitätstheoretiker/innen, nach der die Risikologik «kein Interesse daran hat, Linien zwischen normal und deviant zu ziehen» (Mopas \& VALVERde 2004: 243). Auch wenn die Norm in der Risikologik qua Berechnung erst hergestellt wird und nicht den unhinterfragten Ausgangspunkt bildet, so tut sie in ihrer Anwendung gleichwohl eben dies. Risikodiskurse sind deshalb

«Diskurse über abweichendes Verhalten, in denen die Normativität über vermeintlich rationale Kalküle und individualisierte Verantwortungszuschreibungen unsichtbar gemacht werden» (GROENEMEYeR 2007: 180; Hervorhebung B.B.).

Eben hierin besteht die Entpolitisierung qua Versicherheitlichung, von der eingangs die Rede war. Weil der Massstab «Sicherheit» eine «soziale Konstruktion politischer Ordnungen [ist]» (DALBY 1997: 19f.), entscheidet Letztere, was und wer als Sicherheitsrisiko gilt, und das Politische scheint durch die vermeintlich technischneutrale Art der Berechnung zugleich zu verschwinden. Hierin besteht die mit weitreichenden Folgen einhergehende, spezifische ideologische Leistung der «Versicherheitlichung». Durch sie wird ein Sachverhalt «dem politischen Diskurs und den normalen〉 politischen Spielregeln entzogen» (FuCHS \& KRETSCHMANN 2007: 269), was es dem Staat ermöglicht,

«Kritik Einhalt zu gebieten, Debatten abzuwürgen, bürgerliche Freiheiten einzuschränken und, wenn notwendig, Individuen und Gruppen zu zerstören, die politischen Widerstand gegen das System leisten, das die Unsicherheit überhaupt hervorgebracht hat» (Neocleous 2000b: 13).

Wegen dieser Leistungen bezeichnet Neocleous (2008:4) den derzeit zu beobachtenden Sicherheitsdiskurs als «a semantic and semiotic black hole allowing authority to inscribe itself deep into the human experience». Mit Foucault und Bezug zur materialistischen Staatstheorie betont er, «Sicherheit» diene «to neutralize political action, encouraging us to surrender ourselves to the state» (ebd.). 


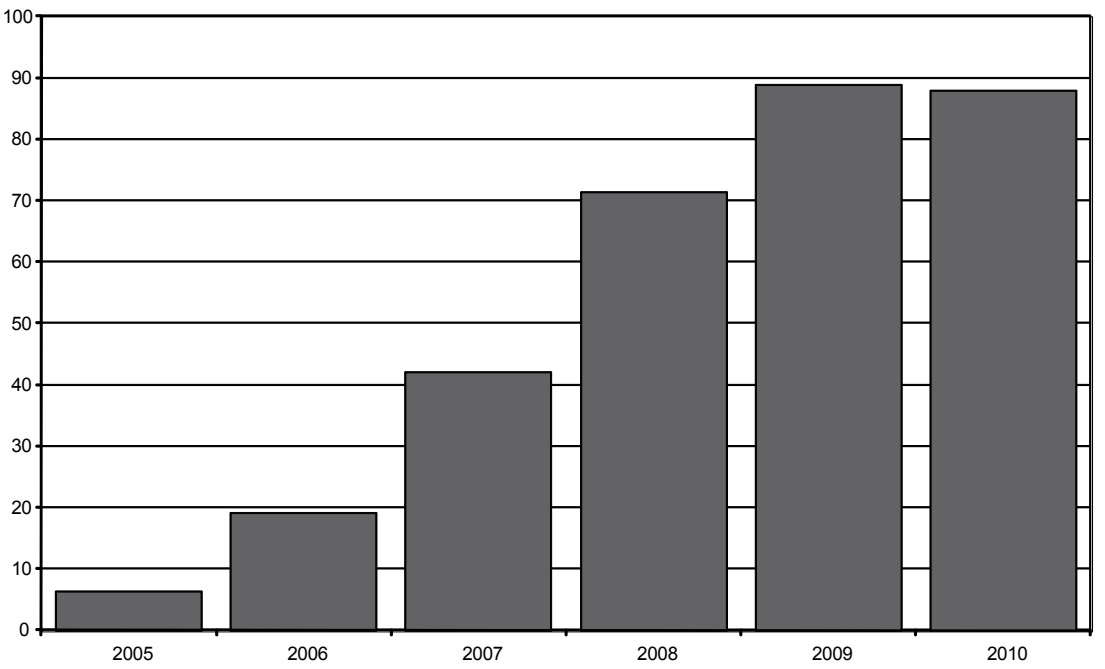

Abb. 1: Budget der EU-Grenzagentur FRONTEX (in Mio. Euro)

Budget of the EU border agency FRONTEX (in millions of Euro)

Le budget de l'Agence européenne pour la gestion de la coopération opérationnelle aux frontières extérieures de l'Union européenne FRONTEX (en millions d'euros)

Quelle: http://www.frontex.europa.eu

\section{Räume des Risikos}

Das «wie» der Versicherheitlichung des EU-Grenzund Migrationsregimes gilt es auf der Basis des «warum» zu klären. Die EU ist im Kern ein gegen die USA gerichtetes imperialistisches Projekt (Altvater \& MAHNKOPF 2007), die in ihr angestrebte und $\mathrm{zu}$ sichernde politische Ordnung dient der polit-ökonomischen Konkurrenz. Dies ist das entscheidende unter den «vielfältigen bestimmenden Elementen» (FouCAULT 1992:37) des Grenz- und Migrationsregimes. Vor diesem Hintergrund werden Grenzüberquerungen als Risiko - weniger unmittelbar für die Akkumulation in der polit-ökonomischen Konkurrenz, als vielmehr für die für jene notwendige politische Ordnung - betrachtet, und es sollen vermeintlich riskante Grenzüberquerende identifiziert und aus dem Territorium der EU herausgehalten werden (vgl. Huysmans 2000). Das angestrebte Migrationsmanagement reagiert als liberale Gouvernementalität darauf, dass «[d]urch Migration [...] die Frage der Bevölkerung kontinuierlich neu gestellt [wird]» (MeYer \& PurTsChert 2008: 150). So sollen nach Plänen der Europäischen Kommission durch den «Einsatz biometrischer Technologie» einerseits «verschärfte Kontrollen» installiert werden, um «Illegale aufzuspüren», andererseits «automatische Abfertigungen» für «〈vertrauenswürdige〉 Reisende» dafür sorgen, dass diese «schnell und ohne lange War- tezeiten durch die Kontrollen gelangen» (BoLESCH 2008). Die Trennung zwischen beiden Gruppen wird mittels Risikokalkulationen eingezogen, Raumproduktionen werden dabei als Mittel eingesetzt.

«Die sozialen Beziehungen, konkrete Abstraktionen, haben keine echte Existenz ausser im und durch den Raum. Ihre Grundlage ist räumlich. Die Verbindung 〈Grundlage - Beziehung bedarf in jedem Einzelfall der Analyse» (LefebVRe 1974: 465).

Eben dies gilt auch für die Versicherheitlichung des EU-Grenz- und Migrationsregimes mittels Risikokalkulation. Abschliessend seien einige der Raumproduktionen skizziert, in denen, durch die und mittels derer die soziale Beziehung zwischen dem Grenz- und Migrationsregime der EU auf der einen und den Grenzüberquerer/innen auf der anderen Seite im Kontext der gouvernementalen Risikologik eine «echte Existenz» (ebd.) erlangt, also praktisch und damit real wird.

Die das Territorium der EU begrenzende Linie, die Grenze als «soziologische Tatsache, die sich räumlich formt» (Simmel 2006: 23), ist der primäre so produzierte und dabei «sehr reale, sehr gewaltförmige» (RAJ 2006: 521) Raum des Risikos. Hier findet eine «produktive Verschränkung zwischen Souveränität und Regierung» (MeYer \& PURTSChert 2008: 167) statt, mithin zwischen staatlicher Machtausübung über ein 
Territorium und liberaler Gouvernementalität. Wie ethnographische Forschung mit Kleinhändler/inne/n verdeutlicht, ist der Moment der Kontrolle an der territorialen Grenze nach wie vor entscheidend (BRUNS 2009; EgBert 2006). Die Grenzkontrollen, die durch nationalstaatliche Apparate durchgeführt werden, werden zunehmend durch EU-einheitliche Regularien (Schengen Grenzcodex seit 2006) angeleitet und werden mitunter durch die stetig in ihrer Bedeutung wachsenden EU-Grenzagentur FRONTEX (seit 2004) unterstützt (vgl. Abb. 1). Indem die EU sie so «berechenbar und vergleichbar» (WALTERs 2006:199) macht, wird «ein genuin europäisches Einschluss-Ausschlussverhältnis konstituiert» (BUCKEL \& WISSEL 2009: 390), dessen Ziel darin besteht, «riskanten» Reisenden «selektiv territorialen Zugang zu versagen» (ANDREAS 2003: 79). Dazu verlieren militärstrategische Überlegungen bei der Ausgestaltung der Grenze an Bedeutung, während polizeiliche Tätigkeiten gegenüber Kleinhändler/inne/n, Schmuggler/inne/n und Migrant/ inn/en ins Zentrum rücken (ANDREAs 2003).

Obschon die territoriale Grenze als Risikogrenze nach wie vor von zentraler Bedeutung ist, stellt ein zentrales Kennzeichen des EU-Grenz- und Migrationsregimes dessen Exterritorialisierung dar. Zunehmend wird versucht, die Einreise in die EU bereits vor der territorialen Grenze zu regulieren (und häufig zu verhindern), wobei «Sicherheit» stets stärker gewichtet wird als die Rechte von Flüchtlingen (BUCKEL \& Wissel 2009; Human Rights Watch 2006; LHOMEL 2007). Damit wird die Grenze einerseits delokalisiert (WALTERS 2006: 193-195) und ubiquitär (RAJ 2006: 518f.), andererseits entstehen eben dadurch Orte jenseits der Grenze, in denen der Umgang mit dem Risiko in Form von Migrant/inn/en, Flüchtlingen und anderen Grenzüberschreiter/inne/n konkret wird. Hierzu gehören die Lager inner- und ausserhalb der EU (Le Courrier des Pays de L'Est 2007; Pieper 2008), die Räume der Verkehrsinfrastruktur - in der BRD etwa das durch die Bundespolizei (ehemals Bundesgrenzschutz; vgl. MAURer 2008) zu kontrollierende «Bahnstreckennetz von über $40.000 \mathrm{~km}$ und mehr als 180 Flugplätze mit internationalem Verkehr» (BundesRat 2007: 13) - oder die Konsulate, in denen Bürger/innen von Nicht-EU-Staaten Einreisevisa beantragen: «The EU considers its visa policies a chief means to select 'worthy> from 〈unworthy> guests» (Krause \& Trauner 2008: 6). Die Staatsangehörigkeit fungiert hier als erster «Risikofaktor», aufgrund dessen Bürger/innen von Staaten, die sich auf der «Negativliste» finden, bei der Gewährung stratifizierter Rechtspositionen (vgl. BUCKEL \& WISSEL 2009: 391f.) besonders schlecht abschneiden. Im Rahmen der Europäischen Nachbarschaftspolitik (vgl. BELINA 2009) werden zwischen der EU und ihren Nachbarstaaten Deals ausgehandelt, in denen Erleichterung für bestimmte Gruppen von Antragssteller/inne/n aus solchen Staaten gewährt werden, die ein Rücknahmeabkommen abzuschiebender Flüchtlinge mit der EU unterzeichnen. Dadurch wird eine weitere Sortierung nach Staatsangehörigkeit - die meisten Ausnahmeregelungen werden bei den Westbalkanstaaten, die wenigsten bei Russland gemacht (KraUSE \& TrAUner 2008: 17) - sowie zusätzlich eine solche unter Bürger/ inne/n desselben Staates vorgenommen. Anhand von Kriterien wie Verwandtschaft, Beruf (kein Visum benötigen etwa Fahrer/innen im Warenverkehr, eingeladene Geschäftsleute, Wissenschaftler/innen oder Künstler/innen) oder der Mitgliedschaft in Religionsgemeinschaften werden 〈riskante〉 von 〈erwünschten〉 Grenzüberschreiter/inne/n geschieden.

\section{Resümee und Ausblick}

Die EU mit ihren Apparaten definiert, welche (potentiellen) Grenzüberschreitenden als Risiko für die Sicherheit der angestrebten imperialistischen Ordnung gelten und durch das Grenzregime entsprechend behandelt werden. Als Mittel hierzu produziert sie «Räume des Risikos» am Rand, ausser- und innerhalb ihres Territoriums. Um deren Funktionsweise und Funktion zu verstehen, habe ich in diesem Beitrag drei Theoriestränge verbunden: FoucaulTs Gouvernementalitätsthese, um das «wie» und die Logik der Versicherheitlichung zu verstehen, die materialistische Staatstheorie, um das «warum» zu erklären, sowie die Produktion des Raums, um die «echte Existenz» (LEFEBVRE 1974: 465) von beiden je konkret unter die Lupe nehmen zu können. Auf diese Weise lässt sich Versicherheitlichung m.E. geographisch und im Bezug auf die sie hervorbringenden und prägenden sozialen Prozesse untersuchen.

\section{Literatur}

Althusser, L. (1977 [1970]): Ideologie und ideologische Staatsapparate. Aufsätze zur marxistischen Theorie. - Hamburg \& Berlin: VSA.

Altvater, E. \& B. Mahnkopf (2007): Konkurrenz für das Empire. Die Zukunft der Europäischen Union in der globalisierten Welt. - Münster: Westfälisches Dampfboot.

Andreas, P. (2003): Redrawing the line. - In: International Security 28, 2: 78-111.

Aradau, C. \& R. van Munster (2007): Governing terrorism through risk. - In: European Journal of International Relations 13, 1: 89-115.

Balibar, É. (1991): Foucault und Marx. Der Einsatz des Nominalismus. - In: EWAld, F. \& B. WALdenFELS (Hrsg.): Spiele der Wahrheit. Michel Foucaults Denken. - Frankfurt am Main: Suhrkamp:39-65.

Bauman, Z. (1997): Flaneure, Spieler und Touristen. 
Essays zu postmodernen Lebensformen. - Hamburg: Hamburger Edition.

Belina, B. (2009): Die ENP - eine imperialistische Politik? - In: Heller, W. \& J. Sallanz (Hrsg.): Die Dobrudscha. Ein neuer Grenzraum der Europäischen Union. Sozioökonomische, ethnische, politisch-geographische und ökologische Probleme. $-=$ Südosteuropa-Studien 76, München: Sagner: 157-172.

Belina, B. \& J. Miggelbrink (2010): Am Ostrand des «wettbewerbsfähigsten Wirtschaftsraums der Welt». - In: Wagner, M. \& W. Łukowski (Hrsg.): Alltag im Grenzland. Schmuggel als ökonomische Strategie im Osten Europas. - Wiesbaden: VS Verlag für Sozialwissenschaften (im Erscheinen).

Bolesch, C. (2008): Schutz vor Visa-Missbrauch. - In: Süddeutsche Zeitung vom 14.2.: 7 .

BRuns, B. (2009): Schmuggel an der polnisch-russischen Grenze. - In: Heller, W. \& M. ArambaşA (Hrsg.): Am östlichen Rand der Europäischen Union. Geopolitische, ethnische und nationale sowie ökonomische und soziale Probleme und ihre Folgen für die Grenzraumbevölkerung. - = Potsdamer Geographische Forschungen 28, Potsdam: Universitätsverlag Potsdam: 161-174.

Buckel, S. \& A. Fischer-Lescano (2007): Einleitung. - In: Buckel, S. \& A. Fischer-Lescano (Hrsg.): Hegemonie gepanzert mit Zwang. Zivilgesellschaft und Politik im Staatsverständnis Antonio Gramscis. - Baden-Baden: Nomos: 11-18.

BuCKEL, S. \& J. WisSEL (2009): Entgrenzung der europäischen Migrationskontrolle. - In: BRUNKHORST, H. (Hrsg.): Demokratie in der Weltgesellschaft $-=$ Soziale Welt, Sonderband 18, Baden-Baden: Nomos: 385-403.

BundesRat (2007): Entwurf eines Gesetzes zur Änderung des Bundespolizeigesetzes und anderer Gesetze. - Bundesrat Drucksache 546/07, http://dipbt.bundestag.de/dip21/brd/2007/0546-07.pdf 19.10.2009.

Buzan, B., WÆVER, O. \& J. DE WILDE (1997): Security. A new framework for analysis. - Boulder (Colo.): Lynne Rienner Publishers.

DaLby, S. (1997): Contesting an essential concept. - In: Krause, K. \& M. Williams (Hrsg.): Critical security studies. Concepts and cases. - Minneapolis: University of Minnesota Press: 3-31.

Demirović, A. (2008): Das Wahr-Sagen des Marxismus: Foucault und Marx. - In: Prokla 38, 2: 179-201.

Demirović, A. (2007): Politische Gesellschaft - zivile Gesellschaft. - In: Buckel, S. \& A. Fischer-Lescano (Hrsg.): Hegemonie gepanzert mit Zwang. Zivilgesellschaft und Politik im Staatsverständnis Antonio Gramscis. - Baden-Baden: Nomos: 21-41.

EgBert, H. (2006): Cross-border small-scale trading in south-eastern Europe. - In: International Journal of Urban and Regional Research 30, 2: 346-361.

EIGMÜLLER, M. (2007): Grenzsicherungspolitik. Funktion und Wirkung der europäischen Aussengrenze. - Wiesbaden: VS Verlag für Sozialwissenschaften.
EwALD, F. (2002): The return of Descartes's malicious demon. - In: BAKER, T. \& D. Simon (Hrsg.): Embracing risk. The changing culture of insurance and responsibility. - Chicago: University of Chicago Press: 273-301. Foucault, M. (2004a): Sicherheit, Territorium, Bevölkerung. - Vorlesung am Collège de France 1977-1978, Frankfurt am Main: Suhrkamp.

Foucault, M. (2004b): Die Geburt der Biopolitik. Vorlesung am Collège de France 1978-1979, Frankfurt am Main: Suhrkamp.

Foucault, M. (2002 [1974]): Die Wahrheit und die juridischen Formen. - In: Dits et Ecrits II, Frankfurt am Main: Suhrkamp: 669-792.

Foucault, M. (1997 [1976]): Der Wille zum Wissen. - Frankfurt am Main: Suhrkamp.

Foucault, M. (1994 [1975]): Überwachen und Strafen. Die Geburt des Gefängnisses. - Frankfurt am Main: Suhrkamp.

FoucAult, M. (1992 [1978]): Was ist Kritik? - Berlin: Merve.

Fuchs, W. \& A. KRetschmann (2007): Legitimationstechniken, Sicherheitspraktiken. - In: Kriminologisches Journal 39, 4: 260-277.

Füller, H. \& N. MarquardT (2009): Gouvernementalität in der humangeographischen Diskursforschung. - In: Glasze, G. \& A. Mattissek (Hrsg.): Handbuch Diskurs und Raum. - Bielefeld: transcript: 83-106.

GARLAND, D. (1996): The limits of the sovereign state. - In: British Journal of Criminology 38: 445-471.

Giddens, A. (1996): Konsequenzen der Moderne. - Frankfurt am Main: Suhrkamp.

GoRdon, C. (1991): Governmental rationality. - In: Burchell, G., Gordon, C. \& P. Miller (Hrsg.): The Foucault Effect. Studies in governmentality. - London: Wheatsheaf: 1-51.

Gramsci, A. (1992): Gefängnishefte. Band 4. - Hamburg: Argument.

Groenemeyer, A. (2007): Gibt es eigentlich noch abweichendes Verhalten? - In: Kriminologisches Journal 39, 3: 162-184.

Harvey, D. (2007): The Kantian roots of Foucault's dilemmas. - In: Crampton, J. \& S. Elden (Hrsg.): Space, knowledge and power. Foucault and geography. - Aldershot: Ashgate: 41-47.

Hudson, R. (2006): On what's right and keeping left: or why geography still needs Marxian political economy. - In: Antipode 38: 374-395.

Human Rights Watch (2006): Managing migration means potential EU complicity in neighboring states' abuse of migrants and refugees. - http://hrw.org/backgrounder/eca/eu1006/eu1006web.pdf 12.10.2009.

Huysmans, J. (2000): The European Union and the securitization of migration. - In: Journal of Common Market Studies 38, 5: 751-777.

Jessop, B. (2007a): Althusser, Poulantzas, Buci-Glucksmann. - In: Buckel S. \& A. Fischer-Lescano (Hrsg.): Hegemonie gepanzert mit Zwang. Zivilgesellschaft 
und Politik im Staatsverständnis Antonio Gramscis. - Baden-Baden: Nomos: 43-65.

Jessop, B. (2007b): Kapitalismus, Regulation, Staat. Ausgewählte Schriften. - Hamburg: Argument.

Kramer, A. (1975): Gramscis Interpretation des Marxismus. - In: Gesellschaft 4: 65-118.

KraUSE I. \& F. Trauner (2008): EC visa facilitation and readmission agreements. $-=$ CEPS Working Documents 290, Brüssel: Centre for European Policy Studies.

Le Courrier des Pays de l'Est (2007): Les centres de rétention en Europe centrale. - No. 1060, mars-avril: 25-37.

Lefebvre, H. (1974): La production de l'espace. - Paris: Edition Anthropos.

Legnaro, A. (2008): Das Projekt Biometrie und das Verschwinden der Unschuld. - In: Kriminologisches Journal 40, 3: 179-199.

LEMKE, T. (2000): Neoliberalismus, Staat und Selbsttechnologien. - In: Politische Vierteljahresschrift 41, 1:31-47. LEMKE, T. (1997): Eine Kritik der politischen Vernunft. Foucaults Analyse der modernen Gouvernementalität. - Berlin: Argument.

Lhomel, E. (2007): Immigration et élargissement de l'Union européenne. - In: Le Courrier des pays de l'Est, No. 1060: 4-24.

Lindner, U. (2006): Staat, Herrschaft, Politik. Zum Verhältnis Poulantzas - Foucault. - In: BretTHAUer, L., Gallas, A., Kannankulam, J. \& I. Stützle (Hrsg.): Poulantzas lesen. - Hamburg: VSA: 154-170.

LINDNER, U. (2008): Antiessentialismus und Wahrheitspolitik. - In: Prokla 38, 2: 203-219.

LinK, J. (2008):Wissen und Macht statt Ideologie und Interesse. - In: Prokla 38, 3: 443-457.

Marti, U. (2008): Kapitalistische Macht und neoliberales Regieren. - In: Prokla 38, 2: 289-305.

MARX, K. (1970 [1844]): Zur Judenfrage. - = MarxEngels-Werke 1, Berlin: Dietz: 347-377.

Marx, K. (1969 [1857]): Einleitung zur Kritik der politischen Ökonomie. - = Marx-Engels-Werke 13, Berlin: Dietz: 613-644

MAurer, A. (2008): Entgrenzung der Bundespolizei. - In: Bürgerrechte \& Polizei/CILIP 90: 21-31.

Meyer, K. \& P. Purtschert (2008): Migrationsmanagement und die Sicherheit der Bevölkerung. - In: Purtschert, P., Meyer, K. \& J. Winter (Hrsg.): Gouvernementalität und Sicherheit. Zeitdiagnostische Beiträge im Anschluss an Foucault. - Bielefeld: transcript: $149-172$.

Mopas, M. \& M. VAlVerde (2004): Insecurity and the dream of targeted governance. - In: LARNER, W. \& W. WALters (Hrsg.): Global governmentality. - London: Routledge: 233-250.

Neocleous, M. (2008): Critique of security. - Edinburgh: Edinburgh University Press.

Neocleous, M. (2000a): The fabrication of social order. A critical theory of police power. - London: Pluto Press.
Neocleous, M. (2000b): Against security. - In: Radical Philosophy, Heft 100: 7-15.

NoIRIEL, G. (1994): Die Tyrannei des Nationalen. Sozialgeschichte des Asylrechts in Europa. - Lüneburg: Klampen.

PiePer, T. (2008): Die Gegenwart der Lager. Zur Mikrophysik der Herrschaft in der deutschen Flüchtlingspolitik. - Münster: Westfälisches Dampfboot

Poulantzas, N. (2002 [1978]): Staatstheorie. Politischer Überbau, Ideologie, autoritärer Etatismus. - Hamburg: VSA.

Pratt, G. (1994): Poststructuralism. - In: Johnston, R.J., Gregory, D. \& D. SMith (Hrsg.): The dictionary of Human Geography. $-3^{\text {rd }}$ edition, Oxford: Blackwell: 468f.

Purtschert, P., Meyer, K. \& J. Winter (Hrsg.): Gouvernementalität und Sicherheit. Zeitdiagnostische Beiträge im Anschluss an Foucault. - Bielefeld: transcript. RAJ, K. (2006): Paradoxes of the border of Europe. - In: International Feminist Journal of Politics 8, 4: 512-534.

Robinson, W. (1950): Ecological correlations and the behavior of individuals. - In: American Sociological Review 15: 351-357.

Simmel, G. (2006 [1908]): Der Raum und die räumliche Ordnung der Gesellschaft. - In: EIGMÜLLER, M. \& G. Voruba (Hrsg.): Grenzsoziologie. Die politische Strukturierung des Raumes. - Wiesbaden: VS Verlag für Sozialwissenschaften: 15-23.

Singelnstein, T. \& P. Stolle (2008): Die Sicherheitsgesellschaft. Soziale Kontrolle im 21. Jahrhundert. - 2. Auflage, Wiesbaden: VS Verlag für Sozialwissenschaften.

Transit Migration Forschungsgruppe (Hrsg.)(2007): Turbulente Ränder. Neue Perspektiven auf Migration an den Grenzen. - Bielefeld: transcript.

WALTERS, W. (2006): Border/security. - In: European Journal of Social Theory 9, 2:187-203.

\section{Zusammenfassung: Wie und warum Staat Sicherheit produziert. Dargestellt anhand der Versicherheitli- chung grenzüberschreitender Mobilität seitens der EU und der so produzierten Räume des Risikos}

In den Vorlesungen zur Gouvernementalität skizziert Foucault die Art und Weise, in der im modernen Staat «aus der Distanz» regiert wird. Diese wird im Artikel dargestellt, materialistisch «geerdet», und es werden hierauf aufbauend die Begriffe Risiko und Versicherheitlichung diskutiert. Die Tauglichkeit dieser Herangehensweise wird anhand der aktuellen Grenz- und Migrationspolitik der EU illustriert, und es werden die in diesem Kontext produzierten Räume skizziert.

Schlüsselwörter: Gouvernementalität, Staatstheorie, Risiko, EU, Grenze, Raumproduktion 
Summary: The how and why of national security. Creation of spaces of risk in securitization of crossborder mobility in the $\mathbf{E U}$

In his lectures on "governmentality», FoucAult briefly describes how populations in modern states are governed «at a distance». This article looks at FoucAuLT's governmentality approach in the light of materialist state theories, focussing in particular on the concepts of risk and securitization. The paper contextualizes the approach on the basis of current border and migration policies in the EU, paying special attention to the «spaces of risk» that are produced in this context.

Keywords: governmentality, state theory, risk, EU, border, production of space

Résumé: La production de la sécurité par l'Etat. Une illustration par la sécurisation de la mobilité transfrontalière dans l'Union européenne et ses espaces du risque

Dans son analyse de la gouvernementalité, FoucAulT montre comment les populations sont gouvernées «à une certaine distance» dans l'Etat moderne. Cet article discute l'approche de la gouvernementalité de FouCAULT en l'inscrivant dans les théories matérialistes de l'Etat. L'article contextualise cette problématique à l'aide des concepts de risque et de sécurisation. Il illustre les techniques de gouvernementalité du risque et de la sécurisation en faisant référence aux récentes politiques de l'Union européenne en matière de mobilité transfrontalière et de migration, en insistant particulièrement sur les «espaces du risque» produits par ce contexte.

Mots-clés: gouvernementalité, théorie de l'Etat, risque, Union européenne, frontière, production de l'espace

Jun. Prof. Dr. Bernd Belina, Institut für Humangeographie, Goethe-Universität Frankfurt a.M., RobertMayer-Strasse 6-8, D-60325 Frankfurt a.M., Deutschland.

e-mail:

belina@uni-frankfurt.de

Manuskripteingang/received/manucscrit reçu le 23.1.2010

Annahme zum Druck/accepted for publication/accepté pour publication: 30.8 .2010 